Supplementary Material

\title{
Scavenging of Acrolein by Food-Grade Antioxidant Propyl Gallate in a Model Reaction System and Cakes
}

\author{
Jiaqi Wang ${ }^{\dagger}$, Yongling $\mathrm{Lu}^{\dagger}$, Tiesong Zheng ${ }^{\dagger}$, Shengmin Sang ${ }^{\ddagger}$, Lishuang $\mathrm{Lv}^{\dagger *}$
}

\section{Supporting Information}

\section{Figure captions:}

Figure S1. High resolution mass spectrometry of PG-ACR

Figure S2. Mass spectrum (MS/MS) of PG-ACR

Figure S3. ${ }^{1} \mathrm{H}-\mathrm{NMR}$ spectrum of PG-ACR

Figure S4. ${ }^{13} \mathrm{C}-\mathrm{NMR}$ spectrum of PG-ACR

Figure S5. HMBC-NMR spectrum of PG-ACR

Figure S6. HMQC-NMR spectrum of PG-ACR

Figure S7. High resolution mass spectrometry of PG-2ACR

Figure S8. Mass spectrum (MS/MS) of PG-2ACR

Figure S9. ${ }^{1} \mathrm{H}-\mathrm{NMR}$ spectrum of PG-2ACR

Figure S10. ${ }^{13} \mathrm{C}-\mathrm{NMR}$ spectrum of PG-2ACR

Figure S11. HMBC-NMR spectrum of PG-2ACR

Figure S12. HMQC-NMR spectrum of PG-2ACR

Figure S13. DEPT-NMR spectrum of PG-2ACR 


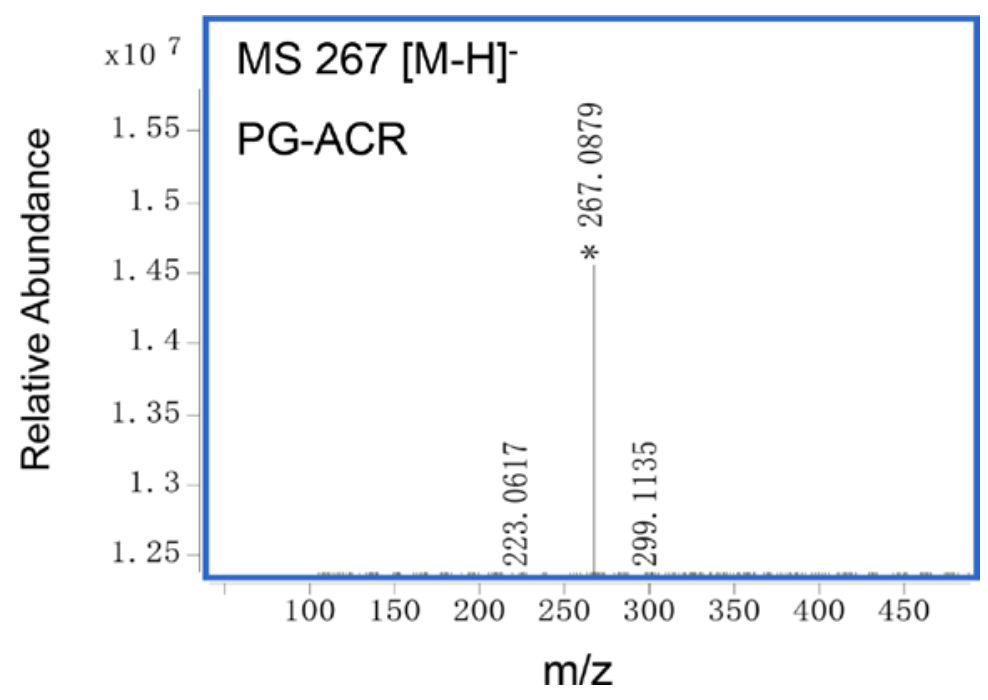

Figure S1. High resolution mass spectrometry of PG-ACR 


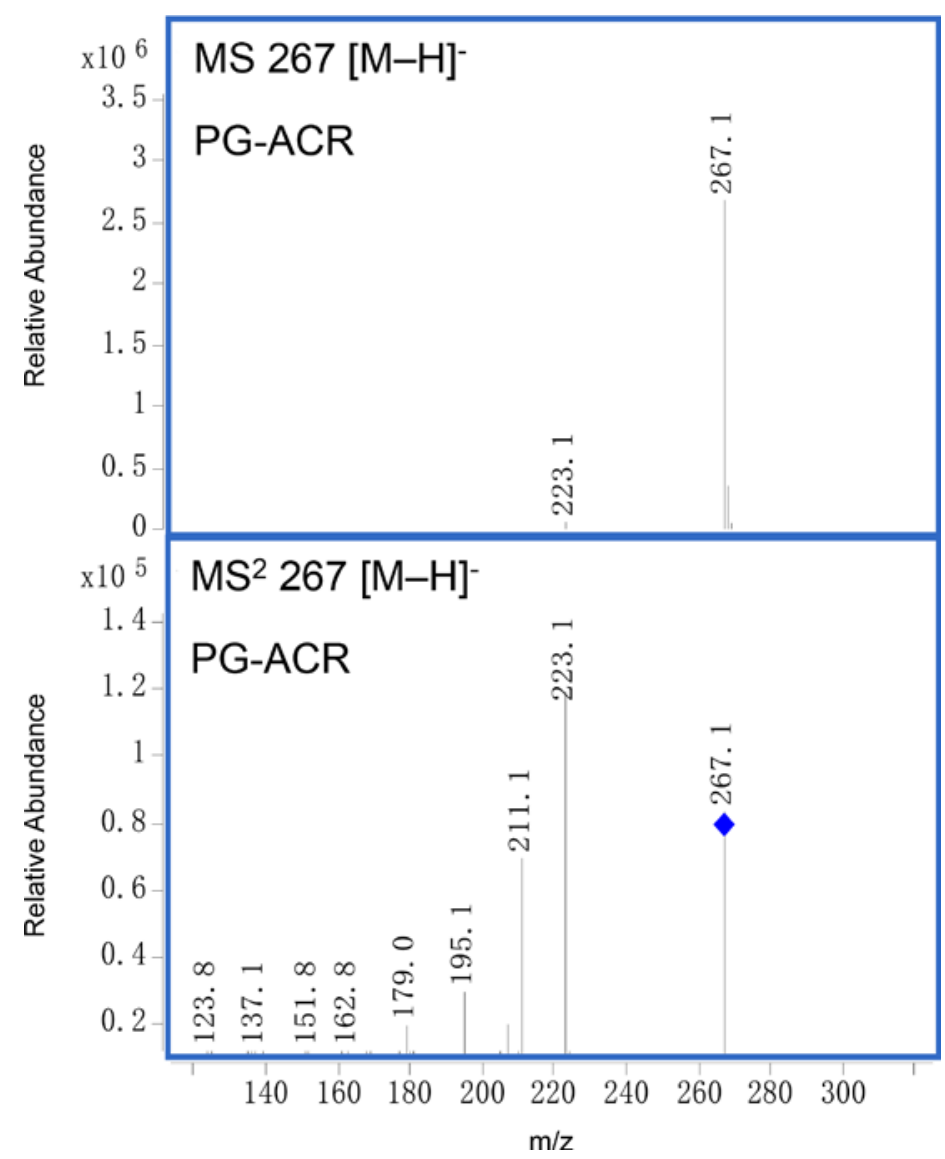

Figure S2. Mass spectrum (MS/MS) of PG-ACR 


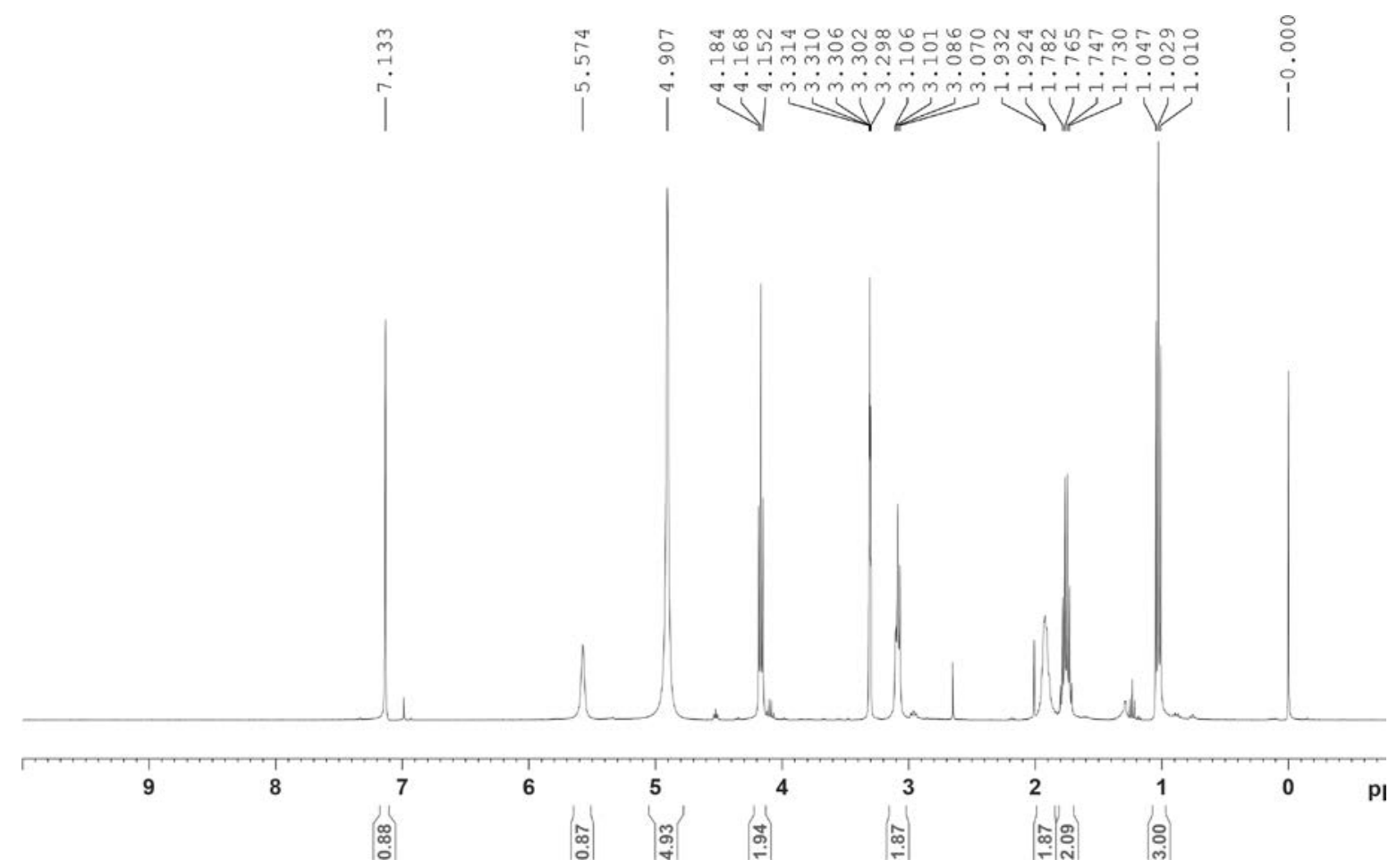

Figure S3. ${ }^{1} \mathrm{H}-\mathrm{NMR}$ spectrum of PG-ACR 


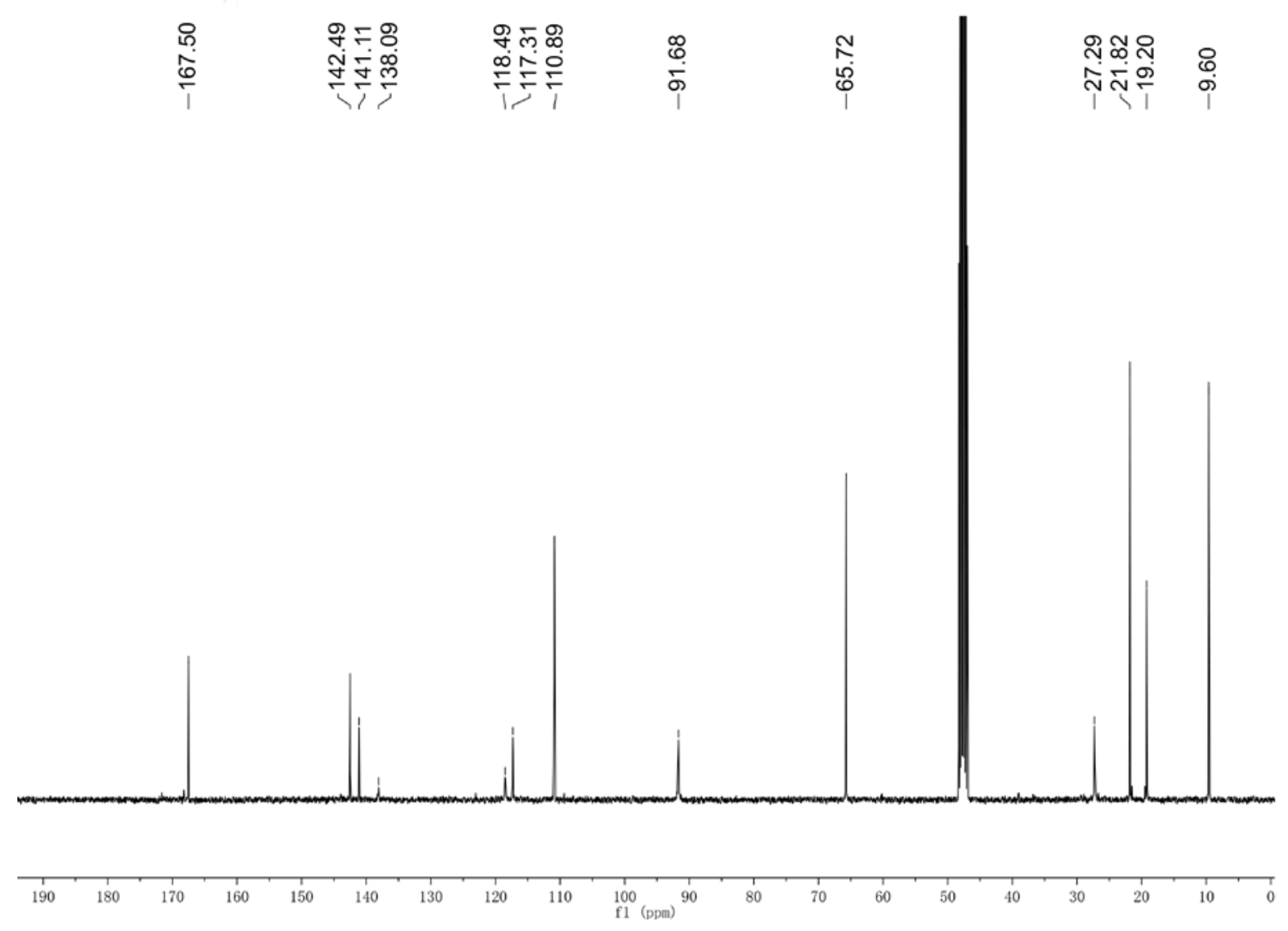

Figure S4. ${ }^{13} \mathrm{C}-\mathrm{NMR}$ spectrum of PG-ACR 


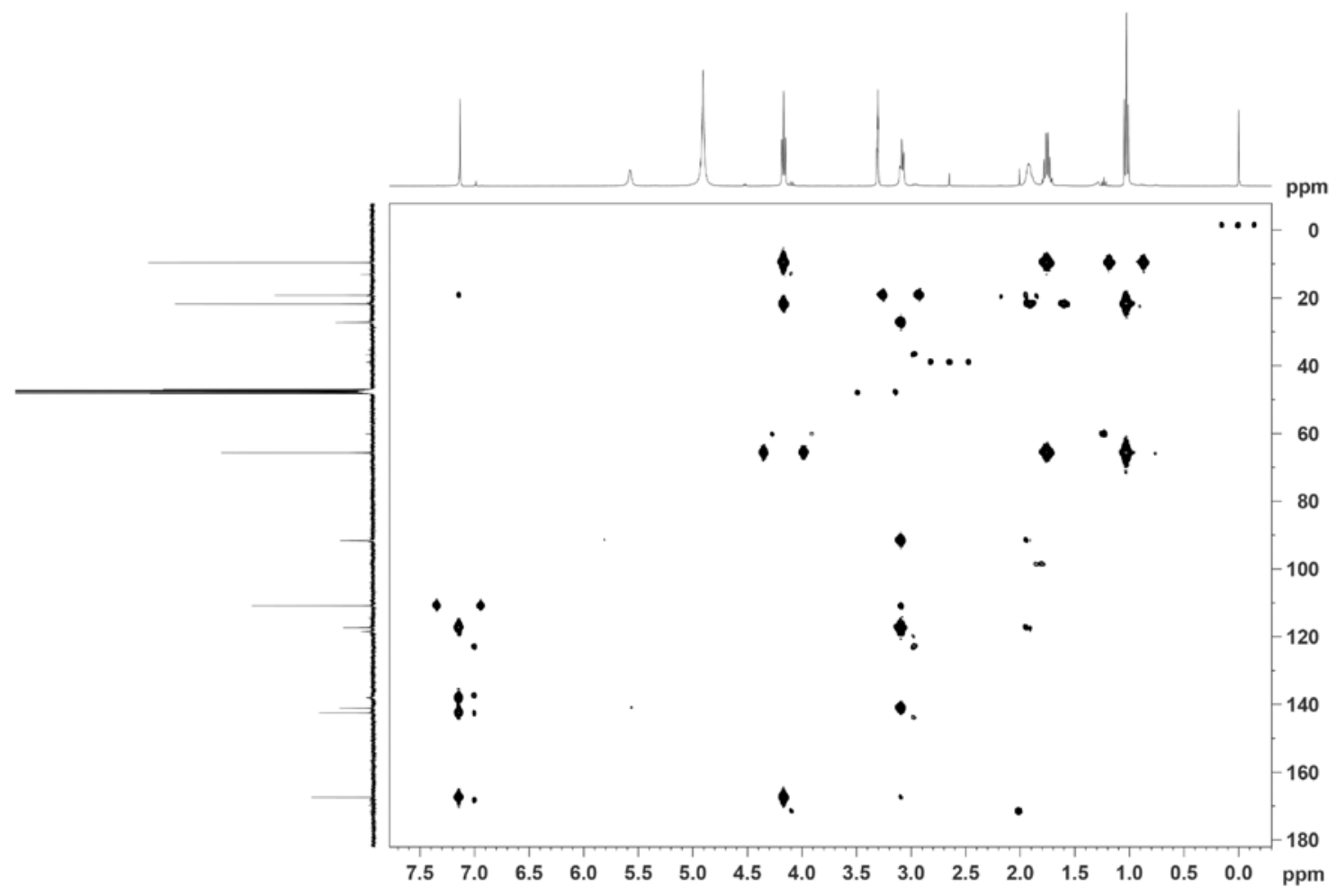

Figure S5. HMBC-NMR spectrum of PG-ACR 


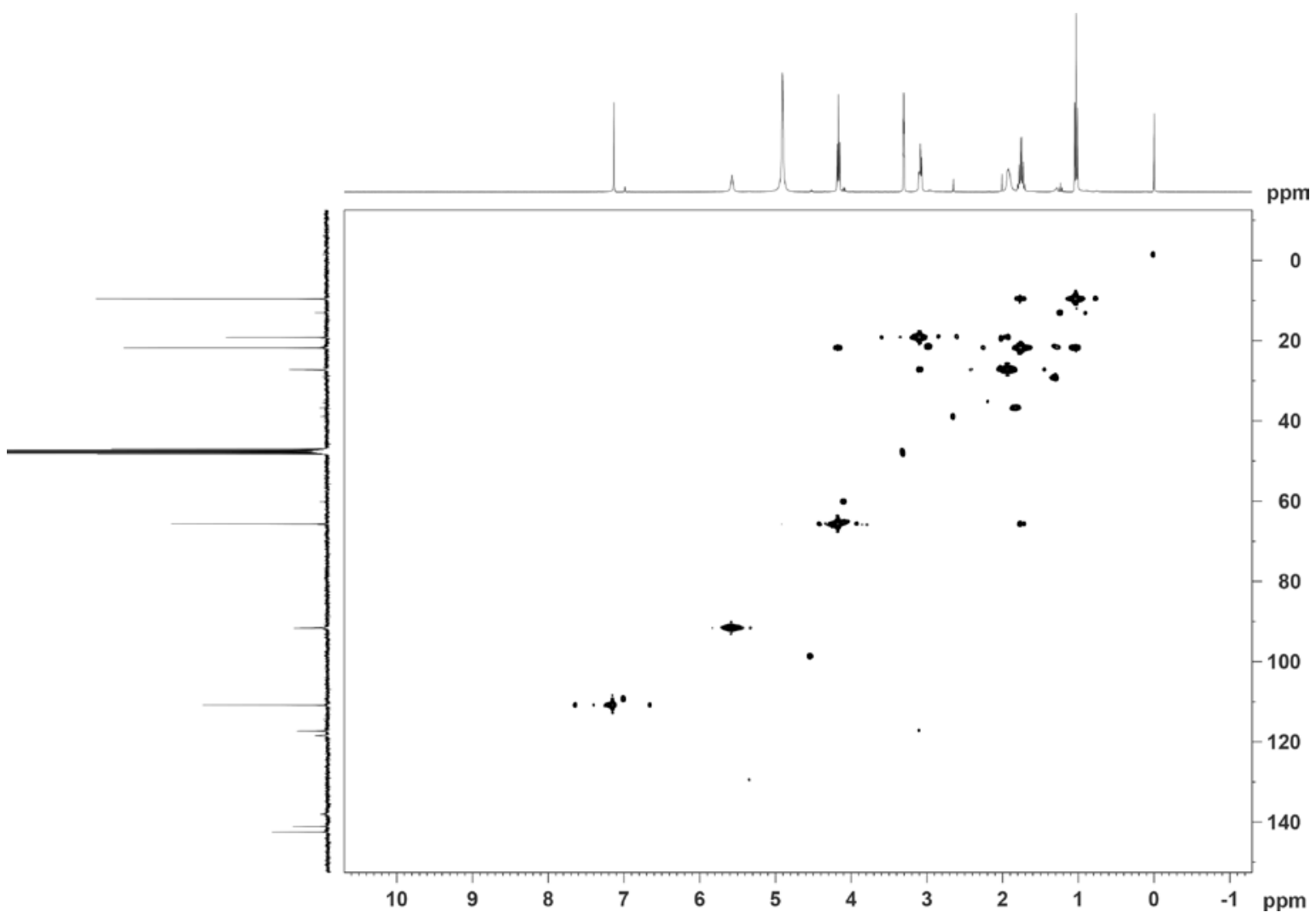

Figure S6. HMQC-NMR spectrum of PG-ACR 


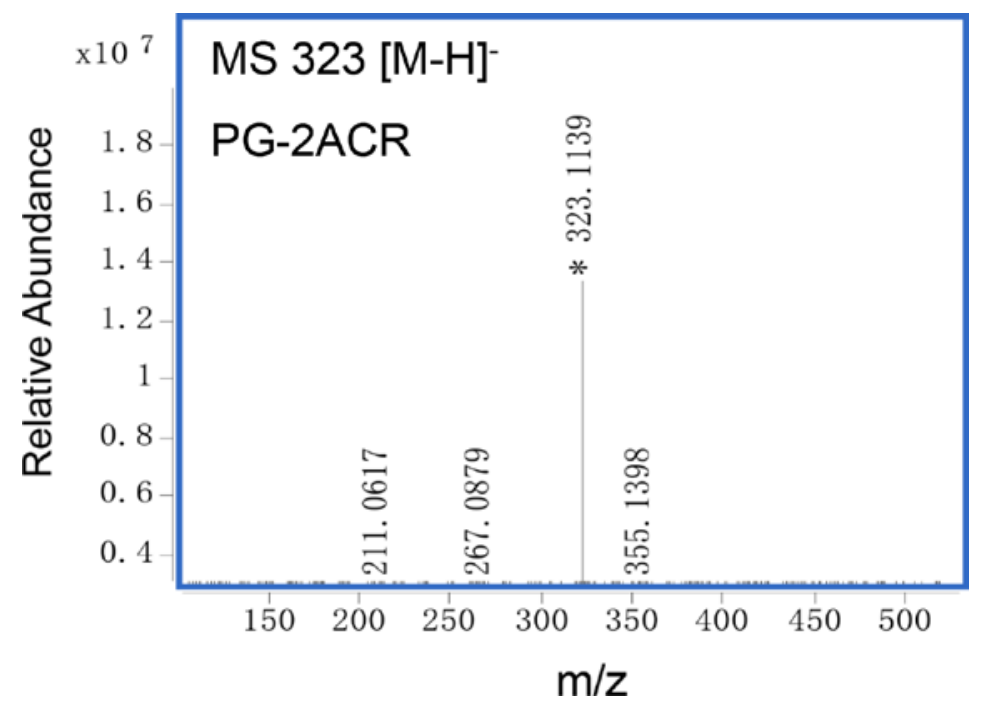

Figure S7. High resolution mass spectrometry of PG-2ACR 


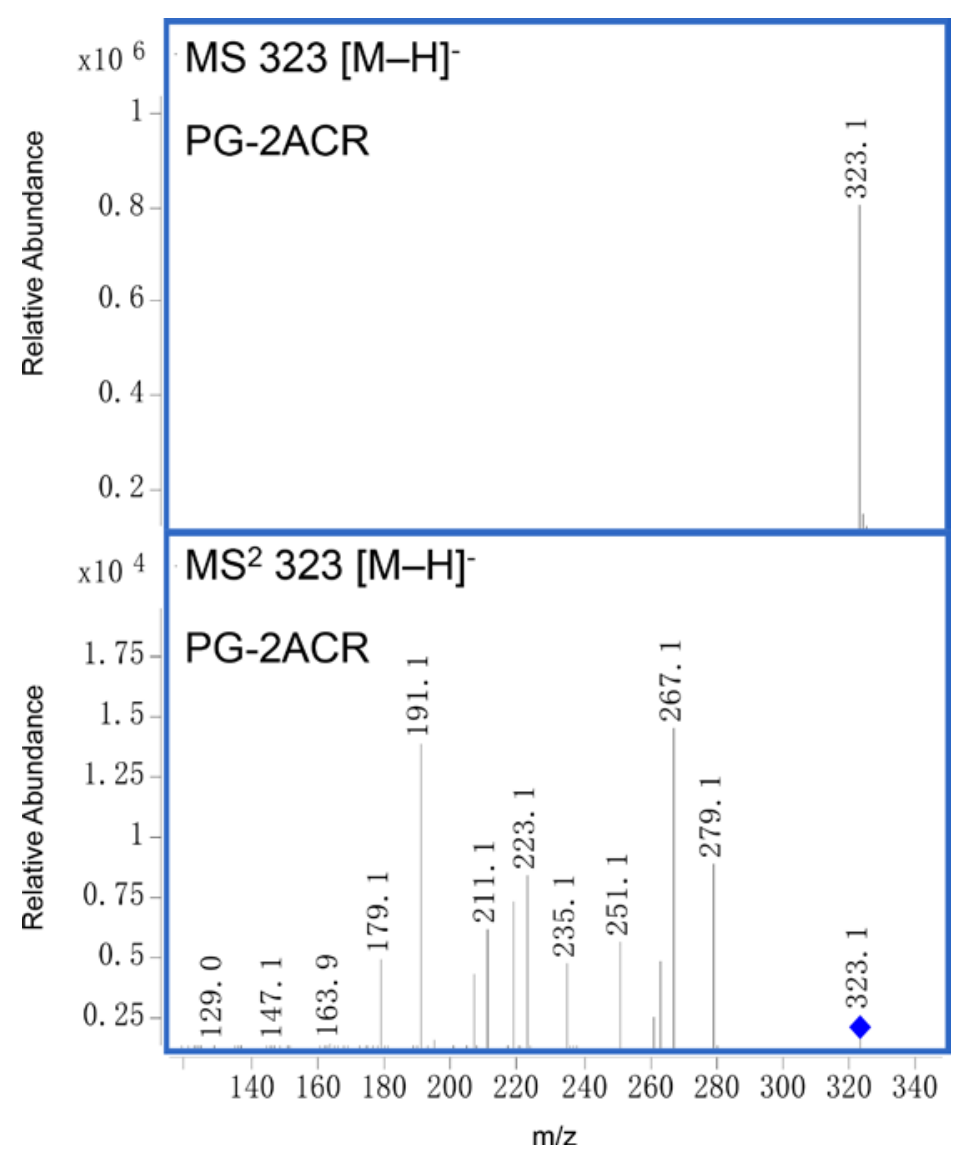

Figure S8. Mass spectrum (MS/MS) of PG-2ACR 


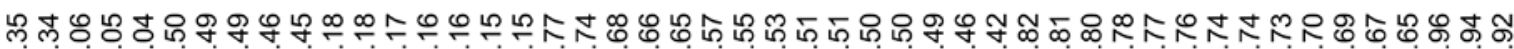

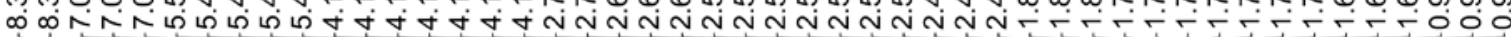

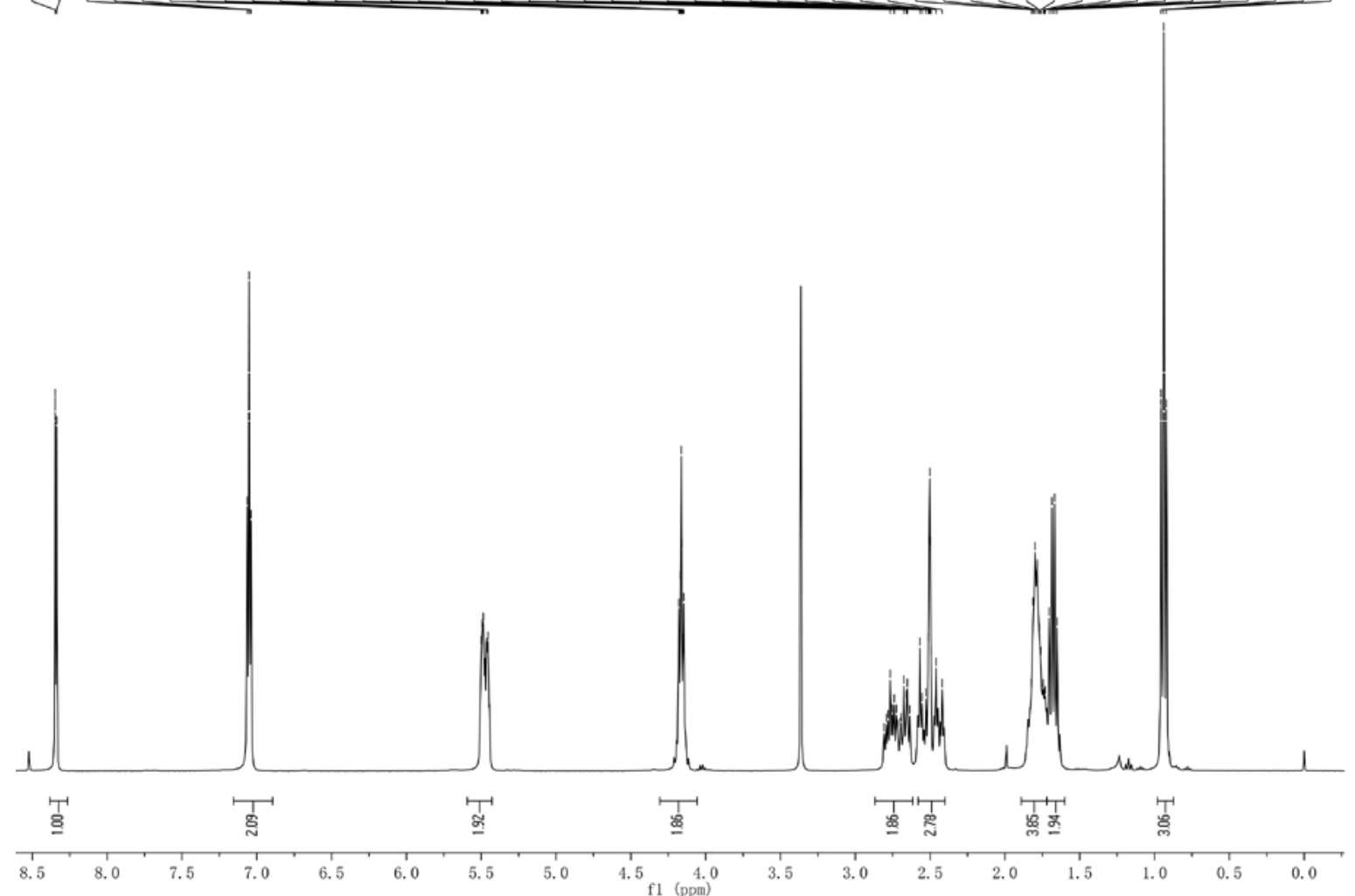

Figure S9. ${ }^{1} \mathrm{H}-\mathrm{NMR}$ spectrum of PG-2ACR 


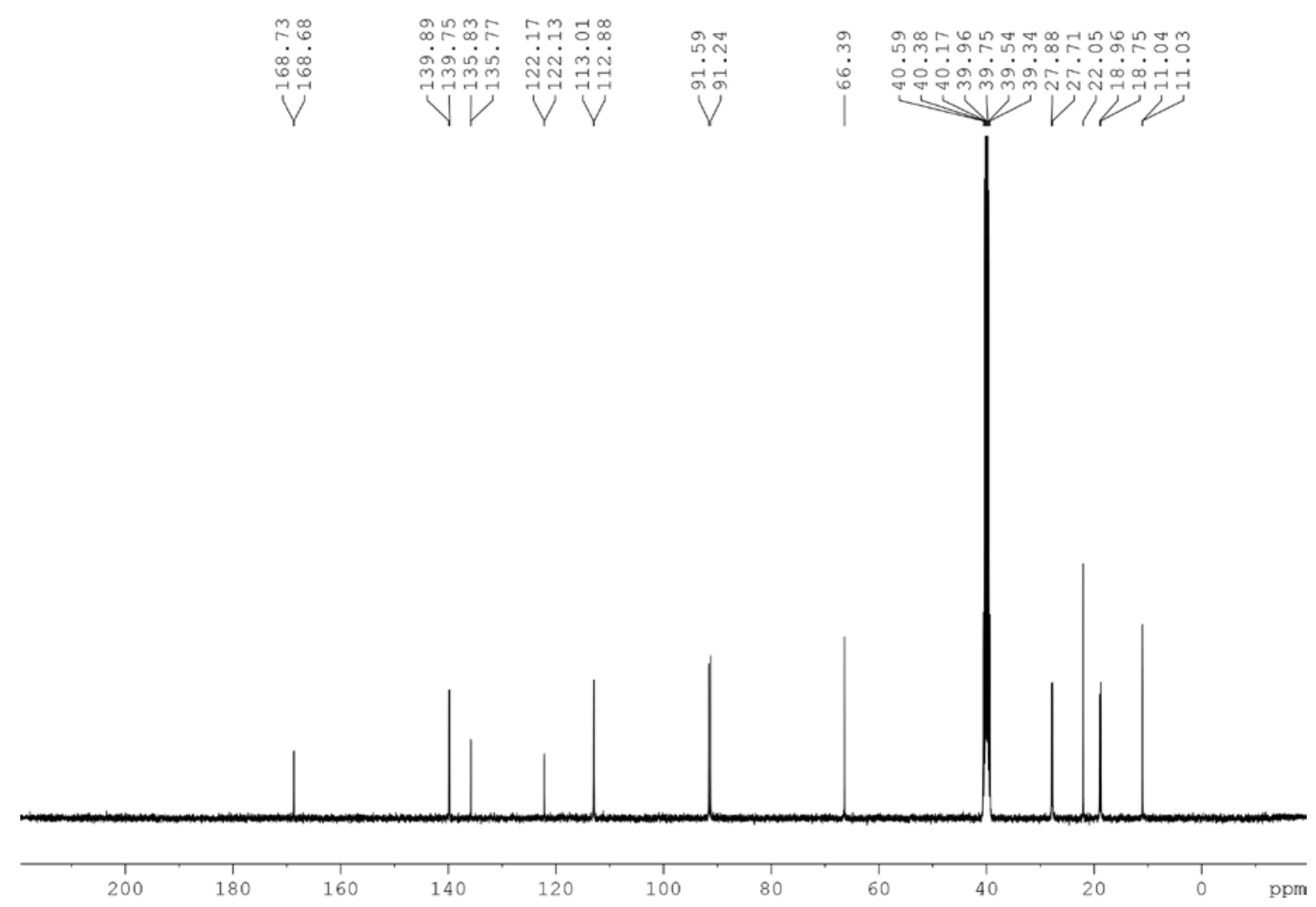

Figure S10. ${ }^{13} \mathrm{C}-\mathrm{NMR}$ spectrum of PG-2ACR 


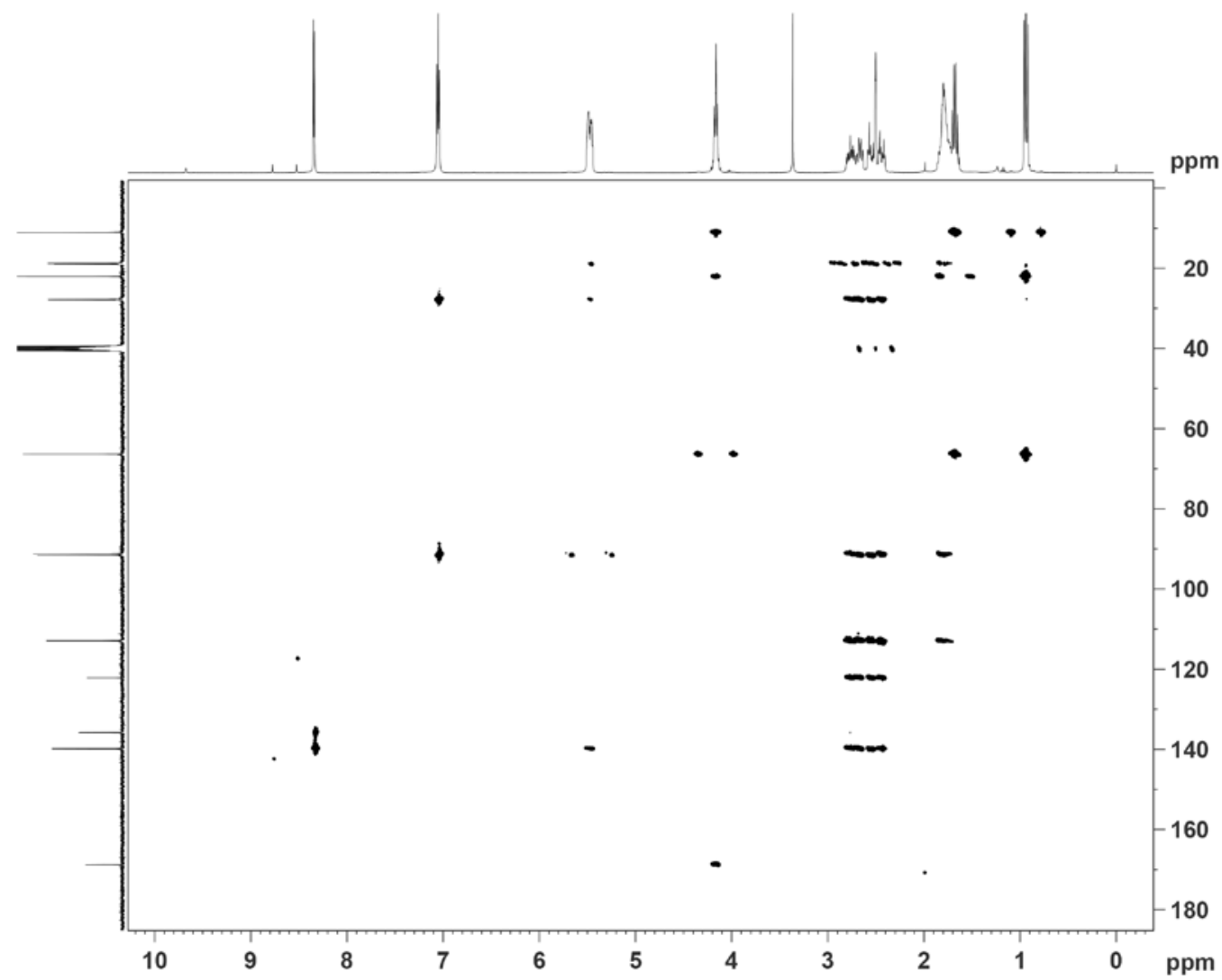

Figure S11. HMBC-NMR spectrum of PG-2ACR 


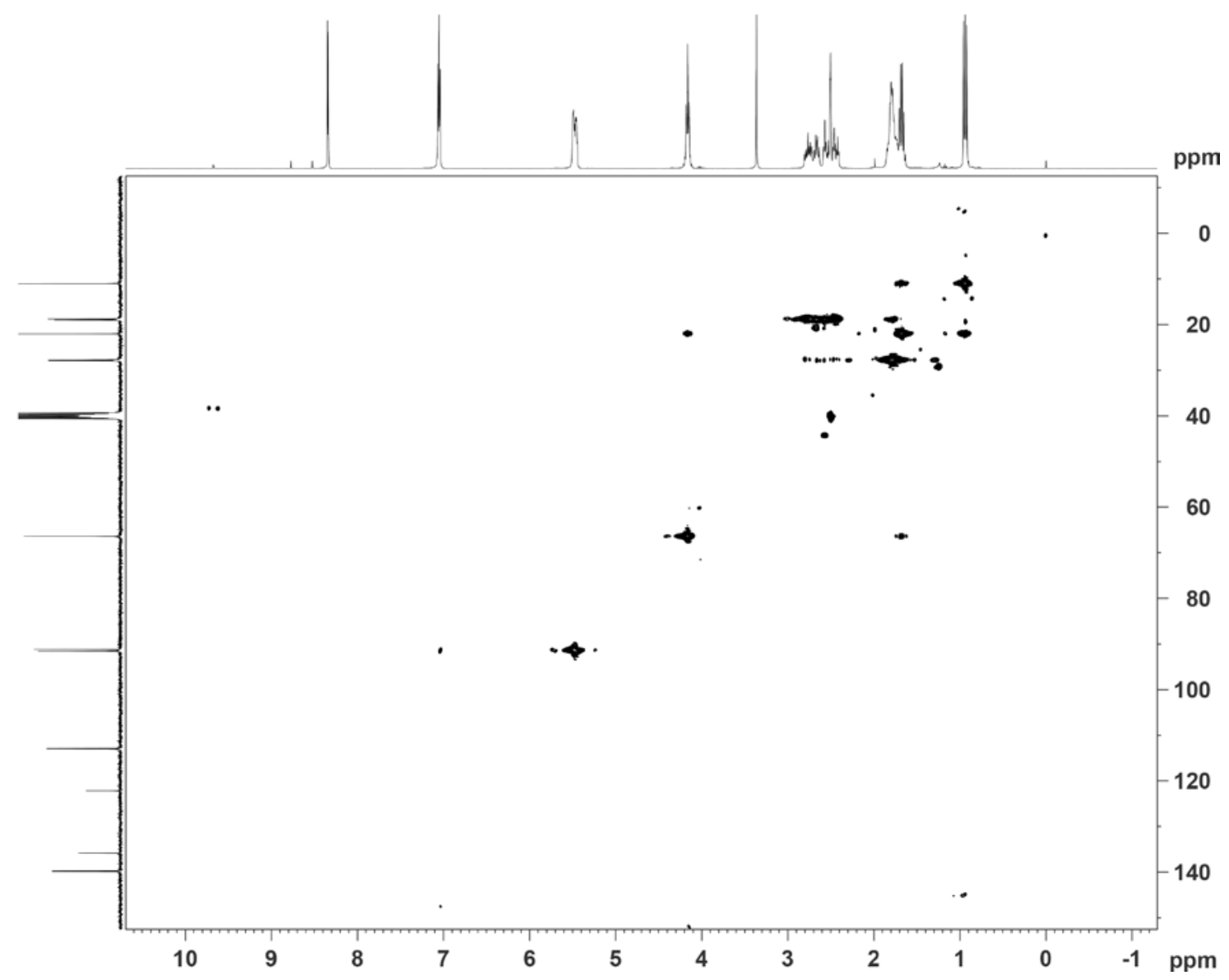

Figure S12. HMQC-NMR spectrum of PG-2ACR 


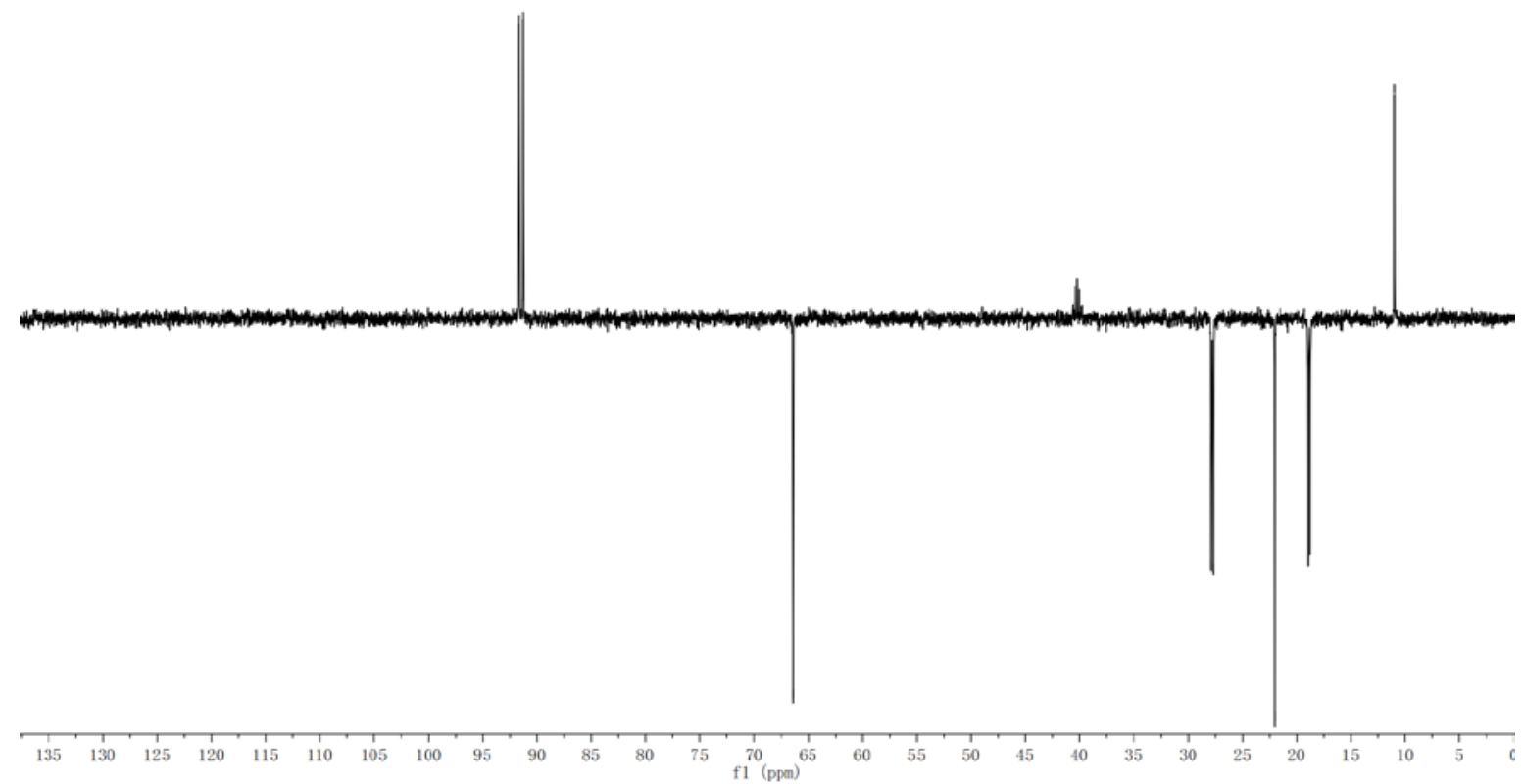

Figure S13. DEPT-NMR spectrum of PG-2ACR 